\title{
The structure of fast sausage waves in current-carrying coronal loops
}

\author{
D. B. Bembitov ${ }^{1}$, B. B. Mikhalyaev ${ }^{1}$, and M. S. Ruderman ${ }^{2,3}$ \\ ${ }^{1}$ Kalmyk State University, Elista, Russia \\ ${ }^{2}$ Solar Physics and Space Plasma Research Centre (SP2RC), School of Mathematics and Statistics, University of Sheffield, \\ Sheffield, UK \\ ${ }^{3}$ Space Research Institute (IKI), Russian Academy of Sciences, Moscow, Russia \\ Correspondence to: B. B. Mikhalyaev (bbmikh@mail.ru)
}

Received: 10 June 2014 - Revised: 28 July 2014 - Accepted: 1 September 2014 - Published: 24 September 2014

\begin{abstract}
We study fast sausage waves in a model coronal loop that consists of a cylindrical core with axial magnetic field and coaxial annulus with purely azimuthal magnetic field. The magnetic field is discontinuous at the tube and core boundaries, and there are surface currents with the opposite directions on these boundaries. The principal mode of fast sausage waves in which the magnetic pressure perturbation has no nodes in the radial direction can exist for arbitrary wavelength. The results for the fundamental radial mode of sausage waves are applied to the interpretation of observed periodic pulsations of microwave emission in flaring loops with periods of a few tens of seconds. Radial plasma motion has opposite directions at the tube and core boundaries. This leads to the periodic contraction and expansion of the annulus. We assume that the principal mode of fast sausage waves in the current-carrying coronal loops is able to produce a current sheet. However, the nonlinear analysis is needed to confirm this conjecture.
\end{abstract}

Keywords. Solar physics, astrophysics, and astronomy (corona and transition region)

\section{Introduction}

It is commonly accepted that a source of energy of simpleloop flares is electric currents that flow from one loop footpoint to the other (Alfvén and Carlqvist, 1967). This concept implies that the flaring loops contain longitudinal currents. At present, there are no accurate observational data on the distribution of the electric current in the loop cross section. There are two different points of view concerning this problem. The first one is the coronal loops can be currentneutral, and the current integrated over the loop cross section is zero. The second one is the coronal loops contain non-zero total electric currents. We consider here the first case.

In application to a magnetic cylinder, the assumption that the total current is zero implies that the tube must consist of at least two regions. We call these regions the core and the annulus. The currents in these regions have opposite directions and equal magnitudes, so the total current is equal to zero. As a result, the external magnetic field is purely axial. We consider here the simplest model of such a magnetic tube. In this model there are only two surface electric currents. This simple model enables us to study linear oscillations analytically.

Fast sausage modes of flaring coronal loops can effectively modulate their microwave and hard X-ray emission by varying a "loss cone" for accelerated electrons (Zaitsev-Stepanov mechanism). These oscillations are observed as quasi-periodic pulsations with periods from subseconds to a few minutes (Zaitsev and Stepanov, 1983; Aschwanden, 1987; Inglis et al., 2008; Nakariakov and Melnikov, 2009). Note that, recently, fast sausage magnetohydrodynamic (MHD) waves have been also observed in the chromosphere (Morton et al., 2012).

An important property of fast sausage modes in a straight homogeneous magnetic tube with the axial magnetic field is that they are trapped waves only when their wavelength is smaller than the cut-off wavelength (see, e.g. Spruit, 1982; Edwin and Roberts, 1983; Cally, 1986). The period and cutoff wavelength of the global sausage mode very weakly depend on the value of the plasma- $\beta$ (Inglis et al., 2009). In a 
case of smooth inhomogeneity of the plasma density in the radial direction, the period of a sausage oscillation always increases with the increase of the longitudinal wavelength, and this dependence saturates in the long-wavelength limit (Nakariakov et al., 2012). Deeper and steeper radial profiles of the Alfvén speed correspond to more efficient trapping of sausage modes: the cut-off value of the wavelength increases with the steepness and the density contrast ratio. The sausage mode damping due to leakage directly depends on the density ratio of the internal and external media with the damping rate declining for higher density contrasts (Nakariakov et al., 2012; Farahani et al., 2014). Hence the leaky fast sausage modes can have high quality, which makes them indistinguishable from the trapped modes. Su et al. (2012) suggested that the short quasi-periodic pulsations with periods $P<60$ s were possibly sausage-mode oscillations and the long quasiperiodic pulsations with periods $P>60 \mathrm{~s}$ were the higher harmonics of slow magnetoacoustic waves.

In this article, we study properties of a principal sausage mode and its possible influence on the electric current distribution. The current-carrying coronal loops have unusual physical properties: oscillations on the principal sausage mode have no cut-off and can exist for large periods (Mikhalyaev and Khongorova, 2012; Khongorova et al., 2012). Here we discuss a possibility of a current-sheet formation in the current-carrying coronal loops due to excitation of their sausage-mode oscillations. The article is organized as follows. In the next section we present the model of a complex magnetic flux tube with surface electric currents. In Sect. 3 we describe sausage modes of the tube and present its dispersion curves. In Sect. 4 we study the structure of the principal sausage mode. Section 5 contains the summary of the obtained results and our conclusions.

\section{Model of current-carrying loops}

A magnetic tube consists of the core region with the axial magnetic field and the annulus with the purely azimuthal magnetic field. The external magnetic field is once again axial. Hence, in cylindrical coordinates $r, \varphi, z$ with the $z$ axis coinciding with the tube axis the background magnetic field and plasma density are given by the following expressions (Mikhalyaev, 2005):

$$
\begin{gathered}
\boldsymbol{B}(r)=\left\{\begin{array}{cc}
B_{\mathrm{i}} \boldsymbol{e}_{z}, & r<b, \\
\left(B_{0} / \alpha r\right) \boldsymbol{e}_{\varphi}, & b<r<a, \\
B_{\mathrm{e}} \boldsymbol{e}_{z}, & a<r,
\end{array}\right. \\
\rho_{0}(r)=\left\{\begin{array}{cc}
\rho_{\mathrm{i}}, & r<b, \\
\rho_{0} /(\alpha r)^{2}, & b<r<a, \\
\rho_{\mathrm{e}}, & a<r .
\end{array}\right.
\end{gathered}
$$

Here $a$ is the radius of the tube, $b$ is the radius of the core region, $\alpha, B_{i}, B_{e}$, and $B_{0}$ are constant, and $\boldsymbol{e}_{\varphi}$ and $\boldsymbol{e}_{z}$ are the unit vectors in the azimuthal and axial direction. The magnetic field has to satisfy the equilibrium conditions, which are the conditions of the magnetic pressure balance at the boundaries $r=a$ and $r=b$,

$B_{\mathrm{i}}^{2}=B_{0}^{2} /(\alpha b)^{2}, \quad B_{\mathrm{e}}^{2}=B_{0}^{2} /(\alpha a)^{2}$.

There are surface currents on these boundaries with the components given by

$j_{\varphi}(b)=j_{z}(b)=B_{\mathrm{i}} / \mu_{0}, \quad j_{\varphi}(a)=j_{z}(a)=-B_{\mathrm{e}} / \mu_{0}$,

where $\mu_{0}$ is magnetic permeability of free space. The main feature of this model is the existence of two helical currents flowing from one foot of the loop to the other. They are shown in Fig. 1. It is straightforward to see that the total current in the loop is zero, so the loop is current-neutral.

Parker (1979) considered a quasi-static expansion of twisted magnetic tubes in the solar atmosphere. He showed that, during this process, the magnetic flux in the tube is redistributed in such a way that the azimuthal flux is concentrated at the peripheral part of the tube, and the axial flux in the tube core. As a result a tube consisting of two parts is formed. In the internal or core part of this tube, the magnetic field is predominantly axial, while it is predominantly azimuthal in the annulus encircling the core region. One can expect that the formation of such magnetic flux tubes consisting of two parts is a natural result of the evolution of twisted magnetic tubes transported in the solar atmosphere from the convection zone.

Standing waves in a magnetic tube consisting of two parts have been already studied by Mikhalyaev and Solov'ev (2005) (see also review by Ruderman and Erdélyi, 2009). However in the model of a magnetic tube studied in this paper, the magnetic field was axial both in the core region and annulus. Erdélyi and Carter (2006) and Carter and Erdélyi (2008) studied the wave propagation in a tube consisting of the core with the axial magnetic field and the annulus with the twisted magnetic field.

\section{Sausage modes}

We describe the plasma and magnetic field perturbations by the linearized magnetohydrodynamic equations for ideal cold (i.e. zero-beta) plasmas:

$\rho_{0} \frac{\partial^{2} \boldsymbol{\xi}}{\partial t^{2}}=\frac{1}{\mu_{0}}(\nabla \times \boldsymbol{b}) \times \boldsymbol{B}+\frac{1}{\mu_{0}}(\nabla \times \boldsymbol{B}) \times \boldsymbol{b}$,

$\boldsymbol{b}=\nabla \times(\boldsymbol{\xi} \times \boldsymbol{B})$,

where $\boldsymbol{\xi}$ is the plasma displacement and $\boldsymbol{b}$ the magnetic field perturbation. The background magnetic field $\boldsymbol{B}$ and the plasma density $\rho_{0}$ are given by Eqs. (1) and (2). Sausage waves are described by solutions of these equations that are 


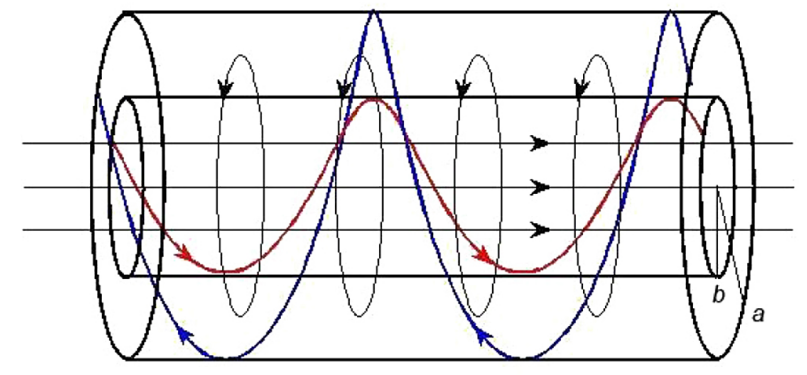

Figure 1. A complex magnetic-flux tube with two surface electric currents. The blue helical curve shows a current line on the external surface of the annulus, while the red helical curve shows a current line on the internal surface of the annulus.

independent of $\varphi$. We Fourier-analysis vectors $\boldsymbol{\xi}$ and $\boldsymbol{b}$ with respect to $z$ and $t$ and write

$$
\begin{gathered}
\boldsymbol{\xi}=\left[\xi_{r}(r) \boldsymbol{e}_{r}+\xi_{z}(r) \boldsymbol{e}_{z}\right] \exp (i k z-i \omega t), \\
\frac{\boldsymbol{b} \cdot \boldsymbol{B}}{\mu_{0}} \equiv p_{T}=p_{T}(r) \exp (i k z-i \omega t),
\end{gathered}
$$

where $p_{T}$ is the magnetic pressure perturbation. Here $\omega$ and $k$ are the frequency and longitudinal wave number. In the core of the tube and in the environment $\xi_{z}=0$, and oscillations are purely radial. The dispersion equation relating $\omega$ and $k$ has been derived by Mikhalyaev and Khongorova (2012) and Khongorova et al. (2012). In Fig. 2 the dispersion curves are shown for the case where $a=2 b$ and $V_{A_{0}}=V_{A_{\mathrm{e}}}=3 V_{A_{\mathrm{i}}}$, where $V_{A_{\mathrm{i}}}, V_{A_{0}}$, and $V_{A_{\mathrm{e}}}$ are the Alfvén speeds in the core of the tube, in the annulus, and in the environment respectively. They are given by

$V_{A_{\mathrm{i}}}=\frac{B_{\mathrm{i}}}{\sqrt{\mu_{0} \rho_{\mathrm{i}}}}, \quad V_{A_{0}}=\frac{B_{0}}{\sqrt{\mu_{0} \rho_{0}}}, \quad V_{A_{\mathrm{e}}}=\frac{B_{\mathrm{e}}}{\sqrt{\mu_{0} \rho_{\mathrm{e}}}}$.

The principal mode (marked by 0 ) in which the magnetic pressure perturbation has no nodes in the radial direction can exist for any wave number. In the thin tube approximation $(k b \ll 1)$, its frequency is described by the approximate expression

$\omega^{2} \approx k^{2} V_{A_{0}}^{2} \frac{\ln (a / b)}{2+\ln (a / b)}$.

Since $\omega / k<V_{A_{\mathrm{e}}}$ the principal mode is a wave trapped by the tube.

It is expedient to compare the results obtained for the magnetic tube with the annulus with those obtained for an untwisted tube. The dispersion equation for such a tube can be found, for example, in Edwin and Roberts (1983). It can be written as $\mathcal{D}(\omega, k)=0$, where $\mathcal{D}(\omega, k)$ is the dispersion

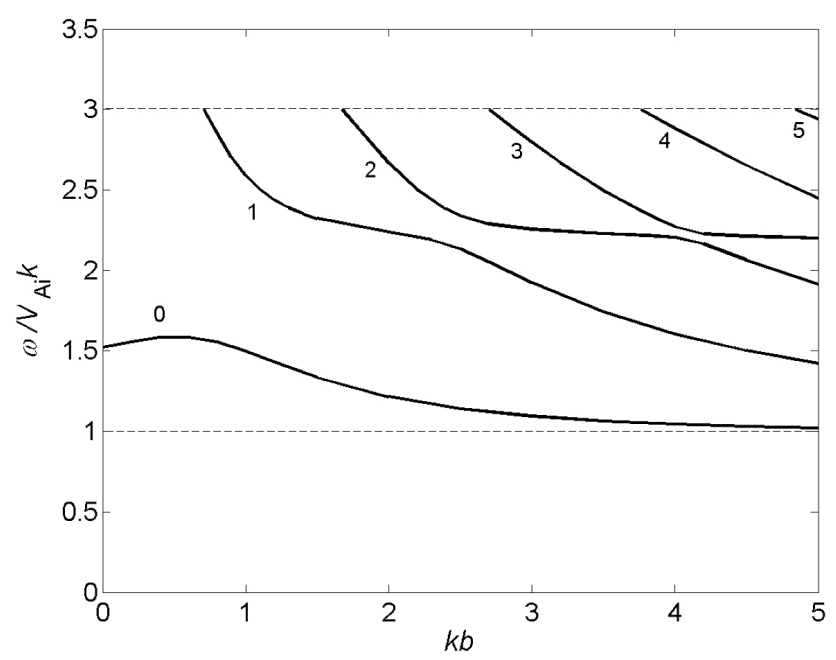

Figure 2. The dispersion curves for fast sausage waves in the complex current-carrying loop with $a / b=2$ and $V_{A_{0}} / V_{A_{\mathrm{i}}}=3$.

function. If we take the limit $b \rightarrow a$ in the dispersion equation for the tube with the annulus derived by Khongorova et al. (2012), then it reduces to $\omega^{2} \mathcal{D}(\omega, k)=0$. What happens with the fundamental mode that, in the case of tube with the annulus, exists for any $k$ ? It follows from Eq. (7) that, in the long wavelength approximation, its frequency tends to zero when $b \rightarrow a$. It turns out that it tends to zero for any $k$, and the zero root in equation $\omega^{2} \mathcal{D}(\omega, k)=0$ just corresponds to this mode. Simultaneously the eigenmode itself tends to zero and, thus, disappears.

The theory of fast sausage waves in current-carrying coronal loops can be applied for interpretation of the observed microwave emission modulation with periods from a few tens of seconds to a few minutes (Khongorova et al., 2012).

\section{Magnetic-tube oscillations}

Let us consider a standing mode of a coronal loop that is fundamental in the longitudinal direction, so that $k=\pi / L$, where $L$ is the length of the loop. We assume that the magnetic field lines are frozen in the dense photospheric plasma at the ends of the tube. For typical flaring coronal loops, $L=$ $30, b=1, a=2 \mathrm{Mm}, V_{A_{\mathrm{i}}}=1000 \mathrm{~km} \mathrm{~s}^{-1}$, and $V_{A_{0}}=V_{A_{\mathrm{e}}}=$ $3000 \mathrm{~km} \mathrm{~s}^{-1}$ (Aschwanden et al., 2004). Then the principal mode dispersion curve gives the wave number $k \approx 0.1 \mathrm{Mm}^{-1}$ and the oscillation period $P \approx 39 \mathrm{~s}$. Such oscillations were observed by Nobeyama Radioheliograph (Kupriyanova et al., 2010).

The dependence of the total pressure perturbation and the radial component of the plasma displacement on $r$ is given by expressions

$p_{T}(r)=\left\{\begin{array}{cc}A_{\mathrm{i}} J_{0}(\kappa r), & r<b, \\ {\left[A_{1} I_{1}(\lambda r)+A_{2} K_{1}(\lambda r)\right] / r,} & b<r<a, \\ A_{\mathrm{e}} K_{0}(\lambda r), & a<r,\end{array}\right.$ 


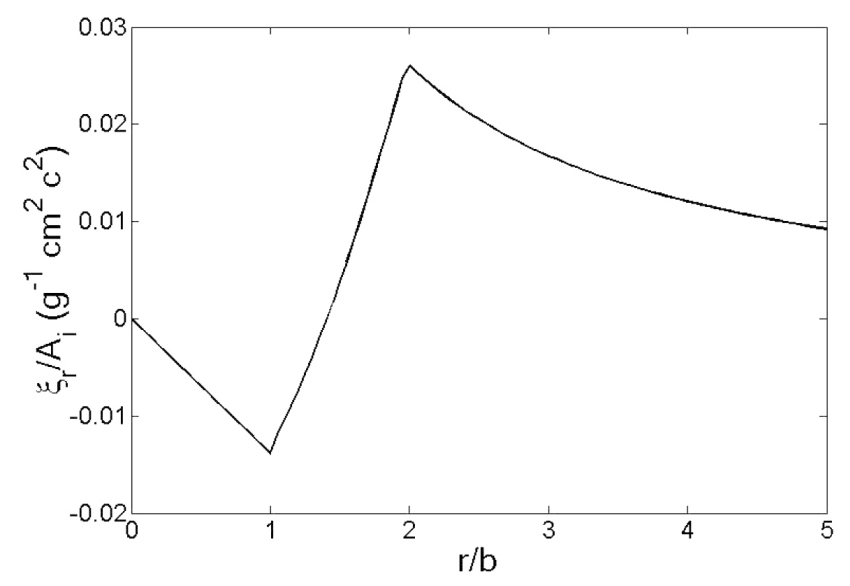

Figure 3. The radial distribution of the radial component of the plasma displacement for the principal sausage mode. Here $a=2 b$.

$\xi_{r}(r)=\left\{\begin{array}{cc}-A_{\mathrm{i}} J_{1}(\kappa r) / \rho_{\mathrm{i}} V_{A_{\mathrm{i}}}^{2} \kappa, & r<b, \\ \frac{\alpha^{2} \lambda r}{\rho_{0} \omega^{2}}\left[A_{1} I_{0}(\lambda r)-A_{2} K_{0}(\lambda r)\right], & b<r<a, \\ A_{\mathrm{e}} K_{1}(\lambda r) / \rho_{\mathrm{e}} V_{A_{\mathrm{e}}}^{2} \lambda, & a<r,\end{array}\right.$

where

$\kappa=\sqrt{\omega^{2} / V_{A_{\mathrm{i}}}^{2}-k^{2}}, \quad \lambda=\sqrt{k^{2}-\omega^{2} / V_{A_{\mathrm{e}}}^{2}}$,

and $A_{\mathrm{i}}, A_{1}, A_{2}$, and $A_{\mathrm{e}}$ are amplitude coefficients that are determined by the boundary conditions. The functions $\xi_{r}(r)$ and $p_{T}(r)$ are shown in Figs. 3 and 4.

The main property of this mode is that the plasma is displaced in the opposite directions in the core of the loop and in the environment. So the excitation of the sausage mode leads to the variation of the loop width, their thickening and shrinkage. For the first half-period the principal sausage mode of our complex magnetic tube leads to shrinkage of the tube and thickening of its core. For the second half-period it leads to thickening of the tube and shrinkage of its core. As a result, surfaces "core annulus" and "annulus environment" with electric currents are periodically pushed toward each other. We assume that realistic coronal magnetic tubes have two co-axial regions with opposite longitudinal diffuse electric currents. The periodic pushing of these two regions toward each other may result in the periodic formation of current sheets. In the case of the fundamental longitudinal mode, the current sheets appear in the middle part of the tube (Fig. 5). This part corresponds to the apex of the coronal loop.

We assume that the principal mode of fast sausage waves in the current-carrying coronal loop is able to produce a current sheet at the apex of the loop.

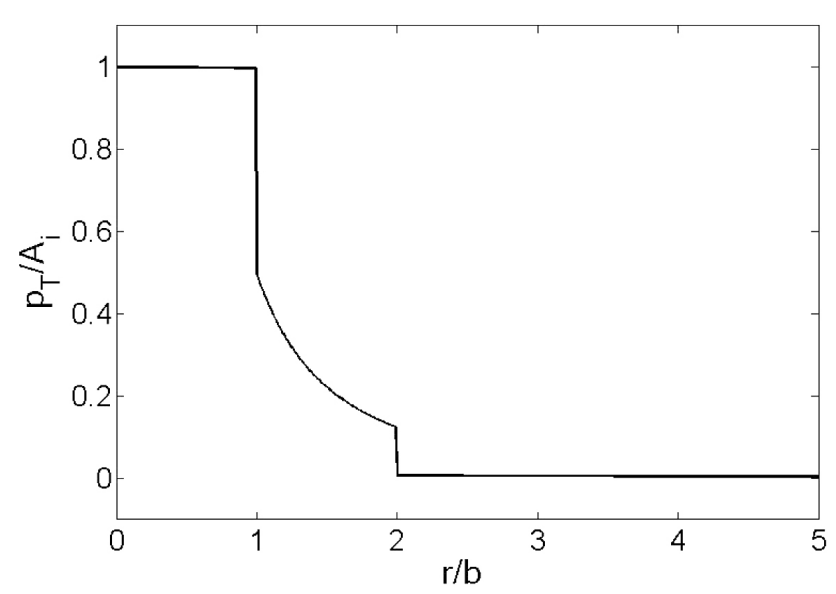

Figure 4. The radial distribution of the total pressure perturbation for the principal sausage mode.

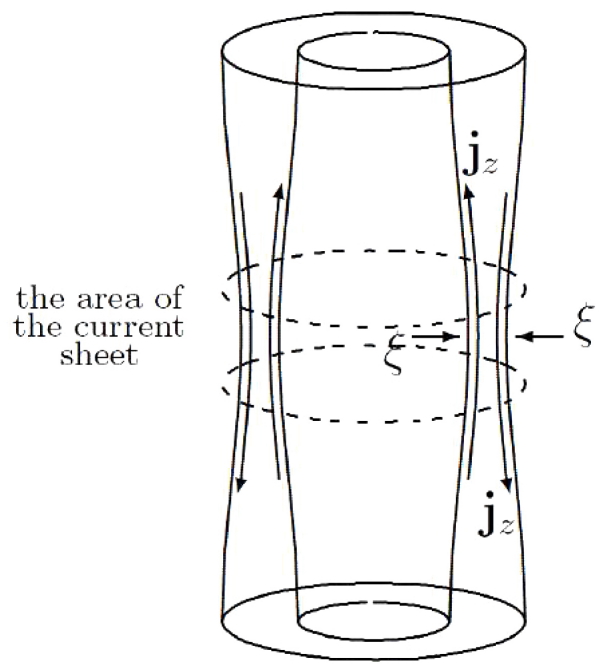

Figure 5. A formation of current sheets at the apex of the coronal loop by its principal sausage mode: $j_{z}$ marks longitudinal electric currents, and $\xi$ marks the plasma displacement.

\section{Conclusions}

The most important properties of the principal sausage mode of the current-carrying coronal loops are the following. Firstly, there is no cut-off frequency in the long-period region. It enables us to describe radio pulsations of flaring loops with periods from tens of seconds to a few minutes. Secondly, in the principal mode the core and the annulus oscillate in the opposite directions. If it remains true for a more realistic model with the diffuse current distribution, then the opposite currents will periodically become closer. When the oscillation amplitude is sufficiently large, oscillations can create current sheets.

We can formulate the problem of nonlinear sausage waves in a magnetic-flux tube containing oppositely directed 
electric currents. If they have the described properties, then we can state that there is a mechanism of the excitation of simple-loop flares. They can be excited due to development of the sausage mode and creation of the current sheet at the loop apex.

This problem has not been studied yet, so we now cannot claim that the hypothetical solution described here exists. However, the results obtained using the simplest model give the ground to hope that the described scenario is possible.

Acknowledgements. This work was supported by the Russian Foundation for Fundamental Research (grant 14-02-00676a). M. S. Ruderman acknowledges the support by the Science and Technology Facilities Council (STFC).

Topical Editor L. Ofman thanks two anonymous referees for their help in evaluating this paper.

\section{References}

Alfvén, H. and Carlqvist, P.: Currents in the solar atmosphere and a theory of solar flares, Solar Phys., 1, 220-228, 1967.

Aschwanden, M. J.: Theory of radio publications in coronal loops, Solar Phys., 111, 113-136, 1987.

Aschwanden, M. J., Nakariakov, V. M., and Melnikov, V. F.: Magnetohydrodynamic sausage-mode oscillations in coronal loops, Astrophys. J., 600, 458-463, 2004.

Cally, P. S.: Leaky and non-leaky oscillations in magnetic-flux tubes, Solar Phys., 103, 277-298, 1986.

Carter, B. K. and Erdélyi, R.: Kink oscillations in magnetic tubes with twisted annulus, Astron. Astrophys., 481, 239-246, 2008.

Edwin, P. M. and Roberts, B.: Wave-propagation in a magnetic cylinder, Solar Phys., 88, 179-191, 1983.

Erdélyi, R. and Carter, B. K.: Wave propagation in incompressible MHD wave guides: the twisted magnetic annulus, Astron. Astrophys., 455, 361-370, 2006.

Farahani, S. V., Hornsey, C., Van Doorsselaere, T., and Goossens, M.: Frequency and damping rate of fast sausage waves, Astrophys. J., 781, 92, doi:10.1088/0004-637X/781/2/92, 2014.
Inglis, A. R., Nakariakov, V. M., and Melnikov, V. M.: Multiwavelength spatially resolved analysis of quasi-periodic pulsations in a solar flare, Astron. Astrophys., 487, 1147-1153, 2008.

Inglis, A. R., Van Doorsselaere, T., Brady, C. S., and Nakariakov, V. M.: Characteristics of magnetoacoustic sausage modes, Astron. Astrophys., 503, 569-575, 2009.

Khongorova, O. V., Mikhalyaev, B. B., and Ruderman, M. S.: Fast Sausage Waves in Current-Carrying Coronal Loops, Solar Phys., 280, 153-163, 2012.

Kupriyanova, E. G., Melnikov, V. F., Nakariakov, V. M., and Shibasaki, K.: Types of microwave quasi-periodic pulsations in single flaring loops, Solar Phys., 267, 329-342, 2010.

Mikhalyaev, B. B.: Rapid damping of the oscillations of coronal loops with an azimuthal magnetic field, Astron. Lett., 31, 406413, 2005 (in Russian).

Mikhalyaev, B. B. and Khongorova, O. V.: Radial oscillations of current-carrying coronal loops, Astron. Lett., 38, 667-671, 2012 (in Russian).

Mikhalyaev, B. B. and Solov'ev, A. A.: The oscillations of coronal loops including the shell, Solar Phys., 227, 249-263, 2005.

Morton, R. J., Verth, G., Jess, D. B., Kuridze, D., Ruderman, M. S., Mathioudakis, M., and Erdélyi, R.: Observations of ubiquitous compressive waves in the Sun's chromosphere, Nat. Commun., 3, A1315, doi:10.1038/ncomms2324, 2012.

Nakariakov, V. M. and Melnikov, V. F.: Quasi-Periodic Pulsations in Solar Flares, Space Sci. Rev., 149, 119-151, 2009.

Nakariakov, V. M., Hornsey, C., and Melnikov, V. F.: Sausage oscillations of coronal plasma structures, Astrophys. J., 761, A134, doi:10.1088/0004-637X/761/2/134, 2012.

Parker, E. N.: Cosmical Magnetic Fields: Their Origin and their Activity, Oxford University Press, London, 237, 1979.

Ruderman, M. S. and Erdélyi, R.: Transverse oscillations of coronal loops, Space. Sci. Rev., 149, 199-228, 2009.

Spruit, H. S.: Propagation speeds and acoustic damping of waves in magnetic flux tubes, Solar Phys., 75, 3-17, 1982.

Su, J. T., Shen, Y. D., Liu, Y., and Mao, X. J.: Imaging observations of quasi-periodic pulsations in solar flare loops with SDO/AIA, Astrophys. J., 755, 113, doi:10.1088/0004637X/755/2/113, 2012.

Zaitsev, V. V. and Stepanov, A. V.: The plasma radiation of flare kernels, Solar Phys., 88, 297-313, 1983. 